\title{
Use of microwave oven improves morphology and staining of cryostat sections
}

\author{
A KENNEDY, A K FOULIS Department of Pathology, Royal Infirmary, Glasgow
}

SUMMARY The quality of microscopic image of cryostat sections that had been subjected to microwave assisted fixation was compared with that resulting from conventional air drying of the sections. The role of microwaves in producing rapid special stains on cryostat sections was also assessed. Methods used permitted stains such as periodic acid Schiff, alcian blue, Gordon and Sweets's reticulin, Masson Fontana, Elastica, Prussian blue and Van Gieson to be performed within three minutes of cutting a cryostat section. The cytological detail of nuclei was much clearer using the microwave technique, allowing more accurate determination of cell type. The microwave oven seems to have major potential in improving the diagnostic accuracy of surgical frozen sections.

The use of cryostat sections in rapid microscopic diagnosis has become a routine part of the histopathological service in most laboratories. The technique is not, however, without its problems. The quality of the microscopic image, particularly when using a high power objective lens, is very poor, and identification of individual cell types, which often relies on good cytological detail of nuclei, is correspondingly difficult. A second problem is that there is usually constraint placed on the time available to make the diagnosis and this by and large precludes the use of histochemical stains other than haematoxylin and eosin.

It has been reported that the microscopic image of a frozen section can be substantially improved by using microwave assisted fixation. ${ }^{1}$ We evaluated this technique to see whether we could confirm this.

Microwave ovens have also been used to allow more rapid staining techniques for formalin fixed paraffin wax embedded sections. ${ }^{2}$ We also assessed the possibility of using a microwave oven to produce rapid special stains on surgical frozen sections.

\section{Material and methods}

The method of Kok et al ${ }^{1}$ was modified as follows. Tissue was mounted on a cryostat chuck with OCT embedding compound (Miles Scientific, Slough, England) and frozen in a slush of methanol and solid carbon dioxide. Five micron sections were cut and picked up on clean glass slides. The cut sections were immediately plunged into $20 \mathrm{ml}$ of $5 \%$ acetic acid in industrial alcohol 990P (Wolman's fixative) in a plastic

Accepted for publication 21 July 1988 five-slide mailer (Histo-Lab, Hertfordshire, England). The slide mailer was placed at the centre of the microwave oven turntable and subjected to eight seconds of microwave irradiation at full power (level $10,600 \mathrm{~W})$ (Network model NW610, Network Electronic Industries, London). The oven was operated at $2.45 \mathrm{GHz}$ and had been designed for domestic use. The lid of the mailer was left open during this period to prevent a build-up of pressure in the container. The sections were then immediately washed in water and rapidly stained with haematoxylin and eosin in the conventional manner used for frozen sections. It was essential that the slide should not dry out before, during, or after the microwave assisted fixation as this was shown to have deleterious effects on nuclear morphological detail.

Section adhesives were not required even for the special staining methods, but the occasional "fatty breast", with a tendency to float, benefited from a reduction in both the power level and the duration of microwaving (to level 1 and five seconds, respectively). The benefit was complemented by transferring the section directly from the microwaved fixative to the haematoxylin solution.

SPECIAL STAINING METHODS

All of the methods listed below could be performed successfully after the acetic-alcohol-microwave fixation protocol. Not all of the steps in any method required microwave stimulation; where this was necessary, the method refers to a standard $(50 \mathrm{ml})$ glass Coplin jar containing the relevant solution. The use of a glass lid to prevent spillage or spurting of solutions is recommended. Glass Coplin jars were preferred to currently available plastic alternatives as they permit- 
ted direct viewing of the staining solution, providing early warning of any solution boiling over. If a solution showed a tendency to boil the method could be changed either by reducing the power level of the machine or reducing the irradiation time.

It should be noted that with reuse of a hot staining solution, much shorter microwave treatments would be required. Fresh solutions used at room temperature worked better, but with the exception of the Perls's potassium ferricyanide-hydrochloric acid reagent, all the staining solutions used in this study were reused several times without apparent deterioration in their staining properties.

\section{Periodic acid Schiff (PAS) ${ }^{3}$}

The standard procedure was adapted by treating the sections with $1 \%$ periodic acid in the microwave oven for 20 seconds at full power. The sections were then rinsed in water and treated with Schiff reagent for 30 seconds at full power. A haematoxylin counterstain was applied after a wash in water, and the sections were dehydrated, cleared, and mounted.

The microwave treatment caused a magenta colouration of the upper layers of the staining solution. This spontaneously disapppeared on standing at room temperature and the solution could safely be reused. If the solution ultimately failed to lose this colour it was discarded.

\section{Alcian blue $-P A S^{3}$}

The conventional $1 \%$ alcian blue in $3 \%$ acetic acid solution was used in the microwave oven at full power for 10 seconds. It was then possible to counterstain with the PAS method described above.

\section{Van Gieson ${ }^{3}$}

The following method did not require microwave stimulation at any stage. Sections were stained with celestin blue for 30 seconds, rinsed in water, and stained in Gill's haematoxylin for 30 seconds. The sections were then rinsed in tap water and blued in Scott's tap water substitute. After a further rinse in tap water the sections were rinsed in methylated spirits and immersed in van Gieson solution for 30 seconds. The sections were then transferred to meths before being dehydrated, cleared, and mounted.

\section{Gordon and Sweets's reticulin ${ }^{3}$}

Only the sensitisation step in $2.5 \%$ iron alum required microwave stimulation (power level 3 for one minute). The acidified potassium permanganate step was limited to 10 seconds before bleaching with oxalic acid. Gold chloride toning was not necessary. Treatment with sodium thiosulphate could also be shortened to 10 seconds without the need for microwaving.

\section{Perls's Prussian blue ${ }^{3}$}

The staining solution was microwaved for 30 seconds at full power. The solution was discarded after use.

\section{Masson Fontana ${ }^{3}$}

The microwave frozen section method differed from the conventional one in that silver solution was used in the microwave oven at power level 5 for one minute. Gold chloride toning was seldom necessary and sodium thiosulphate treatment was limited to 10 seconds without microwaving.

\section{Miller's elastic ${ }^{3}$}

Sections were treated with acidified permanganate for 10 seconds and then bleached with oxalic acid. After rinsing in water the sections were transferred to the staining solution and microwaved for one minute at power level 1. It was convenient to counterstain the sections with van Gieson stain, for which a 10 second dip was adequate.

To make an objective assessment of the quality of image achieved using the microwave assisted frozen section technique, paired sections from 24 specimens (which included 11 different tissues) were processed and stained by the standard technique-frozen sections were warmed, air dried, and then placed in Wolman's fixative 4 - or by the microwave assisted fixation technique. The slides were then viewed blind by four pathologists: they were not told the identity of the pairs. They were asked to state which section of each pair they felt provided the better microscopic image.

\section{Results}

At low power an obvious difference could be discerned between sections which had been treated conventionally (air drying, followed by fixation in Wolman's solution) and those which had been processed using the microwave assisted technique (fig 1). The microwaved sections tended to appear crisper and to have a better definition between haematoxyphilic and eosinophilic areas. The difference in quality of the sections was, however, most obvious when using high power objectives: the cytological detail of nuclei was very much improved by microwaving (fig 2). This difference applied to all tissues tested but was probably most obvious in lymphoid organs. A practical example of the benefit of such improved imaging was a thyroid biopsy specimen for which the differential diagnosis given by the surgeon was Hashimoto's disease or tumour. The frozen section showed a monotonous infiltrate of uniform cells in a collageneous background. On high power examination with the conventional technique the nature of the cells could not be defined and no diagnosis was possible, but the micro- 


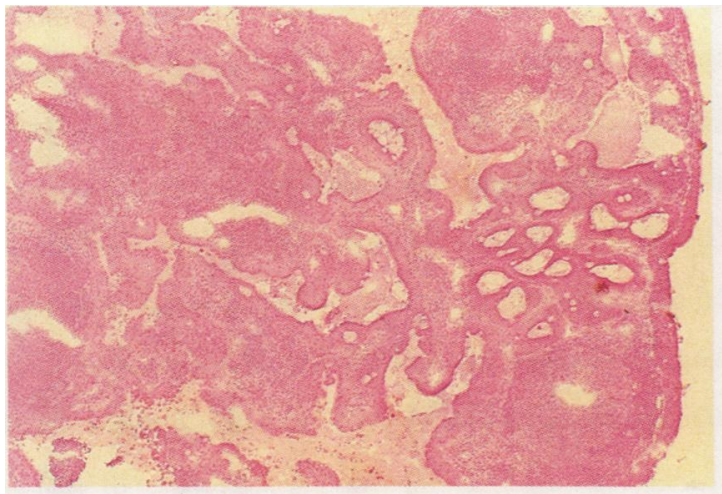

(a)

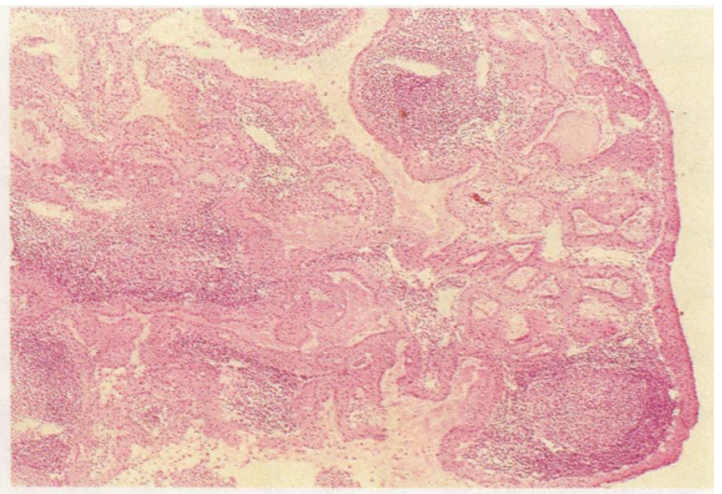

(b)

Fig 1 Cryostat sections of an adenolymphoma of parotid treated conventionally (a) and by microwave assisted fixation technique $(b)$. Note improved low power image in $(b)$. (Haematoxylin and eosin.)

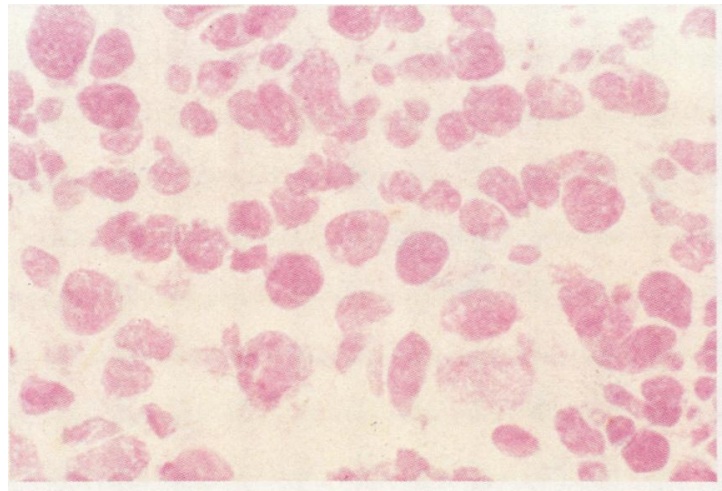

(a)

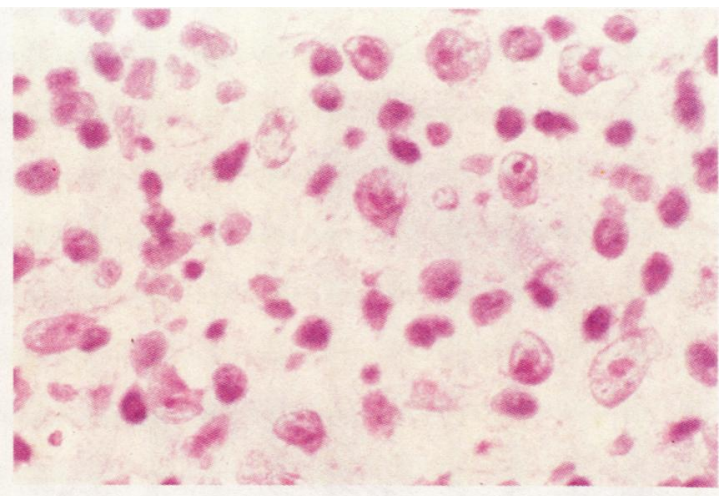

(b)

Fig 2 Cryostat sections of non-Hodgkin's follicular lymphoma treated conventionally (a) and by microwave assisted fixation (b). Note increased nuclear detail (b). Nucleoli are much more prominent and the chromatin pattern approximates more closely to that seen in paraffin wax embedded sections. (Haematoxylin and eosin.)

waved section showed that virtually all the cells were plasma cells and thus Hashimoto's thyroiditis was diagnosed conclusively (fig 3 ).

When the 24 paired sections were given to the four pathologists to determine which sections they thought were more useful diagnostically, 91 of the 96 possible choices favoured the microwaved sections.

All the special stain techniques given here were found to work well on frozen section (figs 4 and 5). All could be performed within three minutes of cutting the frozen section.

\section{Discussion}

Frozen sections have found an established role in the histopathology laboratory. Most pathologists, however, find that when making the diagnosis they have to rely on a relatively low power image because the use of high power objectives adds little information. The poor cytological detail, particularly of nuclei, which generally appear opaque, makes identification of cell types very difficult if their nature was not apparent on the lower power. We can confirm the results of Kok et al ${ }^{\prime}$ : the microscopic image is very much improved when using microwave assisted fixation, and this is bound to lead to greater diagnostic accuracy.

The main purpose of this report has not been to investigate how the microwaving is effective, but it seems likely that microwave irradiation may help in section adherence to the slide. This may be facilitated by microwaves permitting rapid diffusion of water 


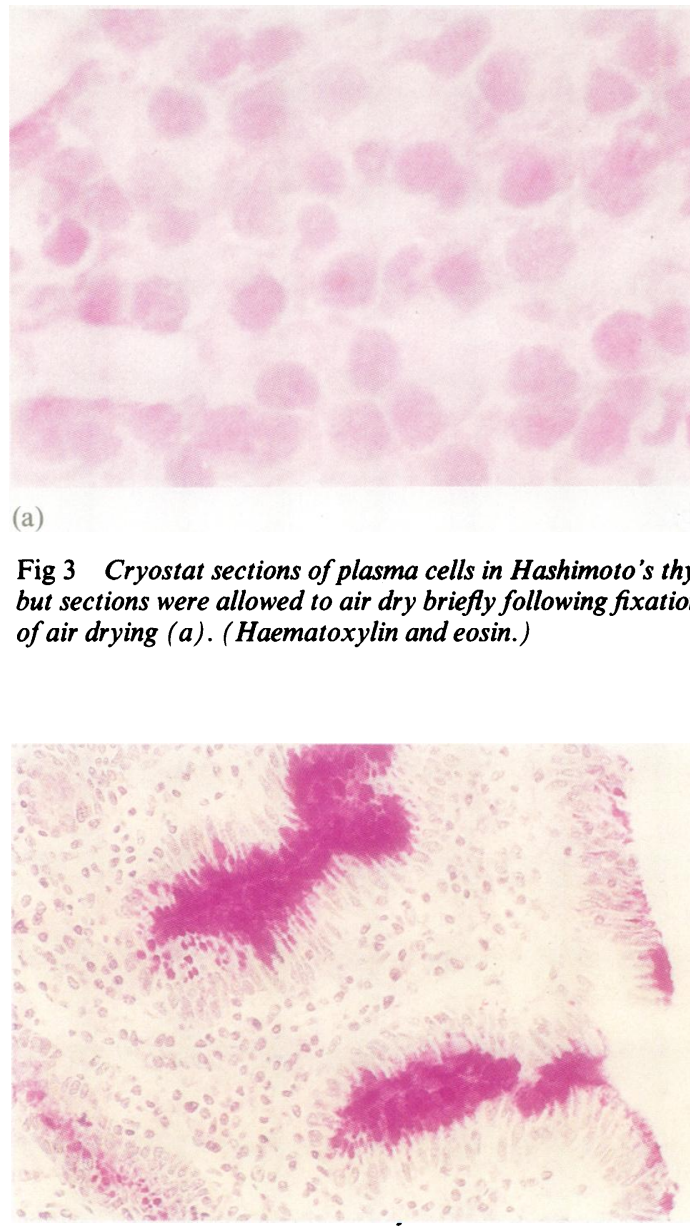

Fig 4 PAS stain on a cryostat section of stomach. This was performed within one minute of cutting the section.

molecules from between the section and the slide. Rapid uniform heat production may also be beneficial. The conventional technique relies on air drying to achieve section adherence before fixation. We have shown that if a section is allowed to air dry, even if it has been microwaved previously, then the quality of the image is poor (fig 3). Thus the improved microscopic image of the microwave assisted fixation technique is probably at least partially due to the ability to avoid the deleterious effects of air drying. No doubt, prompt uniform fixation also helps.

Conventional "special" histochemical stains are seldom used when reporting surgical frozens. We have shown that a wide variety can be performed satisfactorily on frozen sections within three minutes of

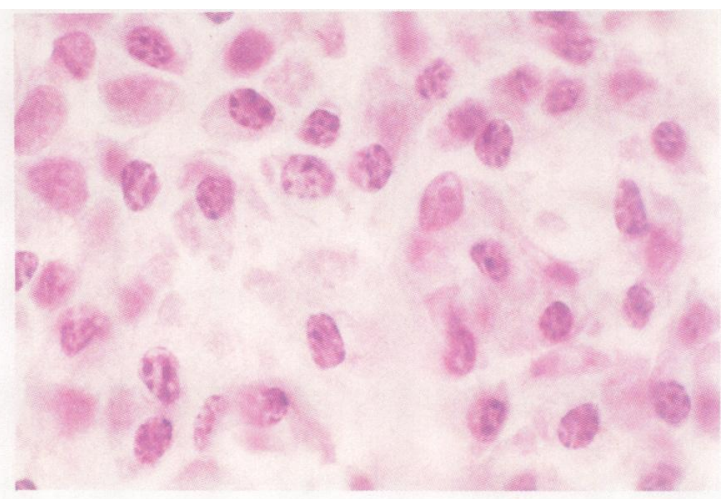

(b) cutting the section, making their use a practical proposition. Individual pathologists will easily be able to imagine situations in which these techniques could be useful. An obvious example would be the use of the PAS method when assessing resection margins in a gastric carcinoma.

The range of methods presented has been restricted to those for which, we believe, there is greatest application in frozen section diagnosis. Our experiments on paraffin wax sections and on frozen sections, however, indicate that virtually any special stain can now be completed within three minutes and can thus be applied on a routine basis to frozen section requests.

Since the techniques described here were developed 
in our laboratory we have abandoned the previous technique of air drying sections. The microwave assisted fixation technique is now also used by other laboratories in our area. Because of the huge domestic market microwave ovens are now cheap. The model used in this study cost $£ 150$. Microwave ovens are finding an increasing number of uses in histopathology laboratories. ${ }^{5}$ We feel that the improvement in quality of frozen sections alone is sufficient reason to purchase such a relatively inexpensive item.

Mrs I Main kindly typed the manuscript.

\section{References}

1 Kok LP, Boon ME, Suurmeijer AJH. Major improvement in microscopic-image quality of cryostat sections combining freezing and microwave-stimulated fixation. Am J Clin :Pathol 1987;88:620-3.

2 Boon ME, Kok LP, Suurmeijer AJH. Microwaves and staining. In: Boon ME, Kok LP, eds. Microwave cookbook of pathblogy. Leiden: Coulomb Press Leyden, 1987:97-114.

3 Bancroft JD, Cook HC. Manual of histological techniques. Edinburgh: Churchill Livingstone, 1984.

4 Culling CFA. Handbook of histopathological and histochemical techniques. Third edition. London: Butterworths, 1974:146-7.

5 Boon ME, Kok LP, eds. Microwave cookbook of pathology. Leiden: Coulomb Press Leyden, 1987:224.

Requests for reprints to: Mr A Kennedy, Department of Pathology, Royal Infirmary, Glasgow, Scotland. 